
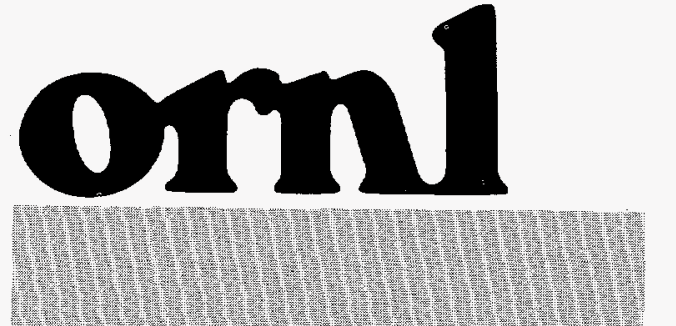

OAK RIDGE NATIONAL LABORATORY

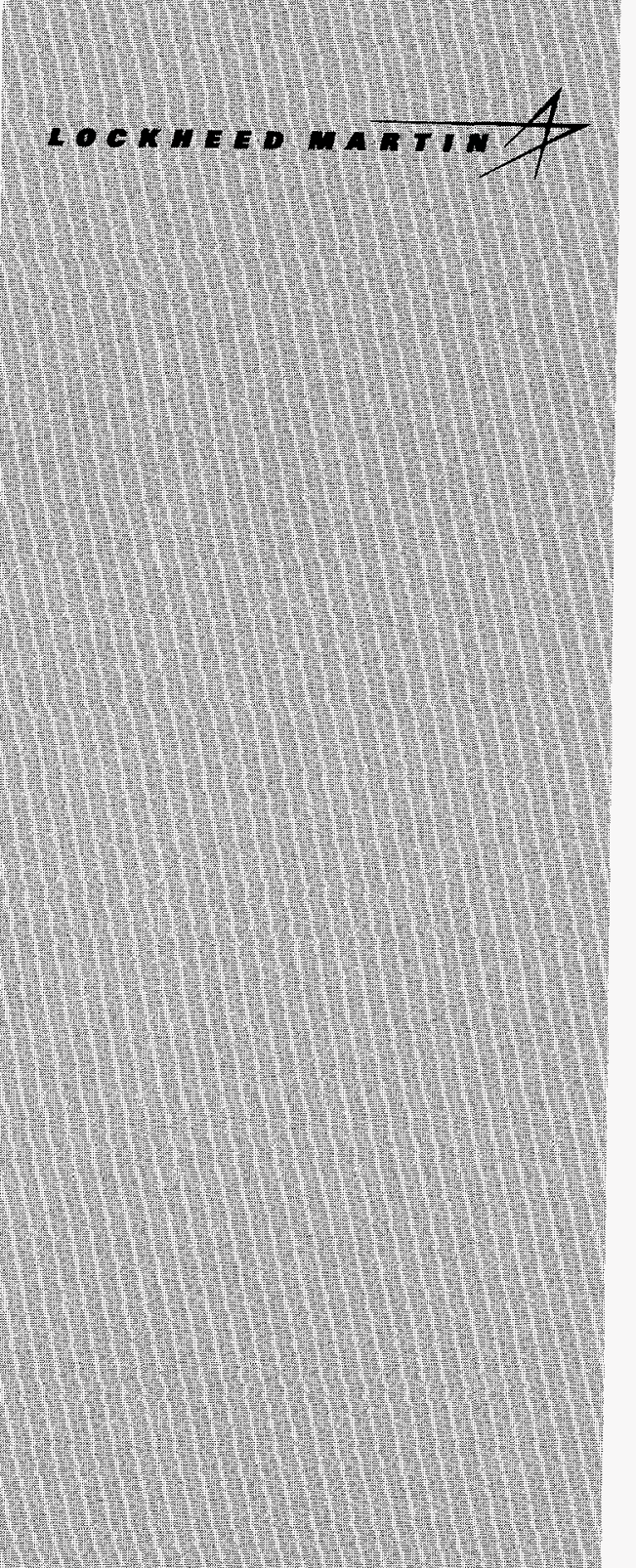

MANAGED ANO CPERATED BY LOCKHEED MARIN ENERGY RESEARCH CORPORATION FORT THE UNTED STATES DEPARMUEMT OF ENERGY

\section{RECEIVED}

\section{AUG 061997 \\ OSTI}

Computer Science and Mathematics Division

Distributed Intelligent Systems Section

\section{THE VELOCITY SNAKE: DEFORMABLE CONTOUR FOR TRACKING IN SPATIO-VELOCITY SPACE}

\author{
Natan Peterfreund \\ Center for Engineering Systems Advanced Research (CESAR) \\ Oak Ridge National Laboratory \\ P.O. Box 2008, Bldg. 6010 \\ Oak Ridge, TN 37831-6355 \\ Email:v4p@mars.epm.ornl.gov
}

Date Published: June 1997

Research supported by the Office of Basic Energy

Sciences, U.S. Department of Energy

Prepared by the

Oak Ridge National Laboratory

Oak Ridge, Tennessee 37831

managed by

Lockheed Martin Energy Research Corporation for the

U.S. DEPARTMENT OF ENERGY

under Contract No. DE-AC05-96OR22464

\section{MASTER 仯}

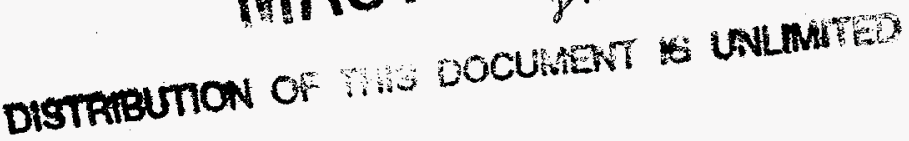


This report has been reproduced directly from the best available copy.

Available to DOE and DOE contractors from the Office of Scientific and Technical Information, P. O. Box 62, Oak Ridge, TN 37831; prices available from (423) 576-8401, FTS 626-8401.

Available to the public from the National Technical Information Service, U.S. Department of Commerce, 5285 Port Royal Road, Springfield, VA 22161.

This report was prepared as an account of work sponsored by an agency of the United States Government. Neither the United States Government nor any agency thereof, nor any of their employees, makes any warranty, express or implied, or assumes any legal liability or responsibility for the accuracy, completeness, or usefulness of any information, apparatus, product, or process disclosed, or represents that its use would not infringe privately owned rights. Reference herein to any specific commercial product, process, or service by trade name, trademark, manufacturer, or otherwise, does not necessarily constitute or imply its endorsement, recommendation, or favoring by the United States Government or any agency thereof. The views and opinions of authors expressed herein do not necessarily state or reflect those of the United States Government of any agency thereof. 


\section{DISCLAIMER}

Portions of this document may be illegible electronic image products. Images are produced from the best available original document. 
Computer Science and Mathematics Division

Distributed Intelligent Systems Section

\title{
THE VELOCITY SNAKE: DEFORMABLE CONTOUR FOR TRACKING IN SPATIO-VELOCITY SPACE
}

\author{
Natan Peterfreund \\ Center for Engineering Systems Advanced Research (CESAR) \\ Oak Ridge National Laboratory \\ P.O. Box 2008, Bldg. 6010 \\ Oak Ridge, TN 37831-6355 \\ Email:v4p@mars.epm.ornl.gov
}

Date Published: June 1997

Research supported by the Office of Basic Energy

Sciences, U.S. Department of Energy

\author{
Prepared by the \\ Oak Ridge National Laboratory \\ Oak Ridge, Tennessee 37831 \\ managed by \\ Lockheed Martin Energy Research Corporation \\ for the \\ U.S. DEPARTMENT OF ENERGY \\ under Contract No. DE-AC05-96OR22464
}




\section{Contents}

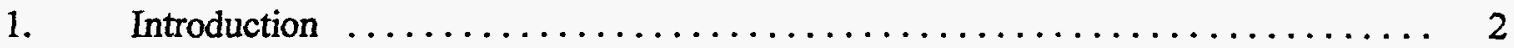

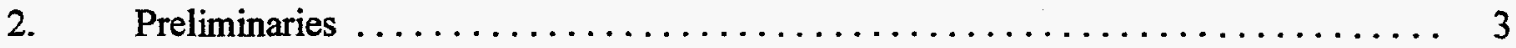

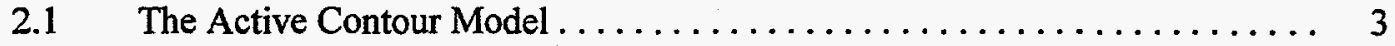

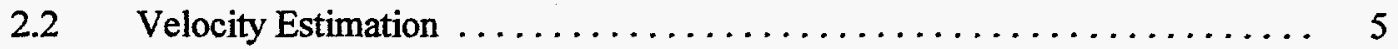

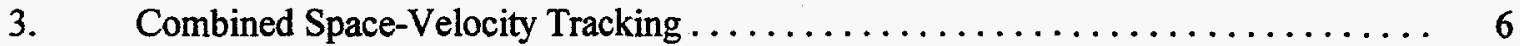

3.1 The Space-Velocity Tracking Model ................... 6

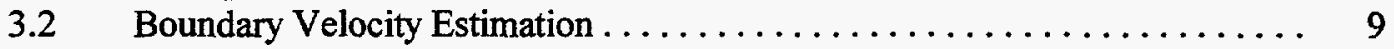

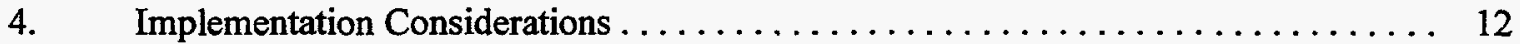

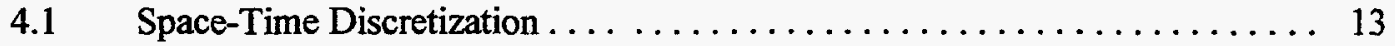

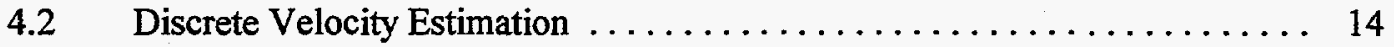

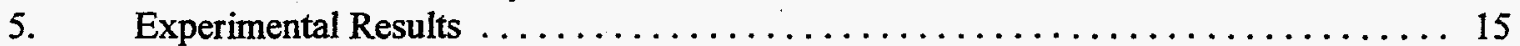

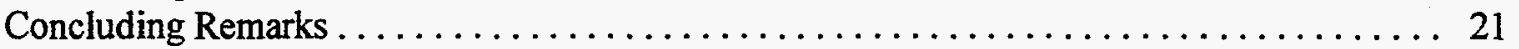

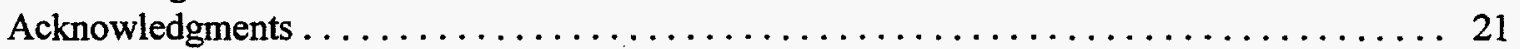

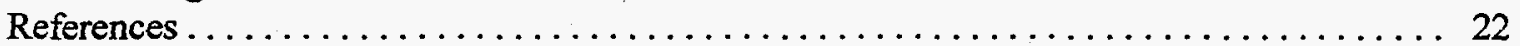

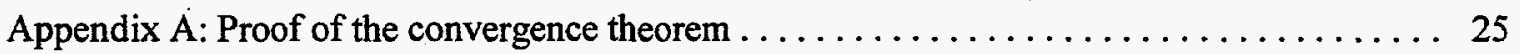




\section{List of Figures}

Figure $1 \quad$ Tracking results of the turning car $\ldots \ldots \ldots \ldots \ldots \ldots \ldots \ldots \ldots \ldots \ldots$

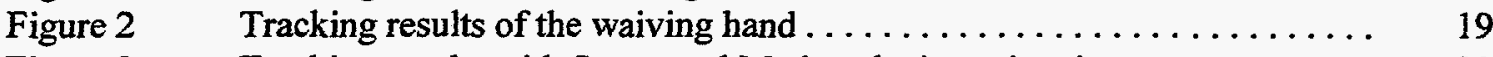

Figure 3 Tracking results with Structured Mode velocity estimation .......... 20

Figure 4 Boundary prediction in the 19-th frame based on the first eight frames .... 20 

. 


\title{
The Velocity Snake: Deformable Contour for Tracking in Spatio-Velocity Space
}

\author{
Natan Peterfreund \\ Center for Engineering Systems Advanced Research (CESAR) \\ Oak Ridge National Laboratory, P.O.Box 2008 \\ Oak Ridge TN 37831-6355. email:v4p@mars.epm.ornl.gov
}

\begin{abstract}
We present a new active contour model for boundary tracking and position prediction of nonrigid objects, which results from applying a velocity control to the class of elastodynamical contour models, known as snakes. The proposed control term minimizes an energy dissipation function which measures the difference between the contour velocity and the apparent velocity of the image. Treating the image video-sequence as continuous measurements along time, it is shown that the proposed control results in an unbiased tracking. This is in contrast to the original snake model which is proven to be biased due to the image (object) velocity, thus resulting in high sensitivity to image clutter. The motion estimation further allows for position prediction of nonrigid boundaries. Based on the proposed control approach, we propose a new class of real time tracking contours, varying from models with batch-mode control estimation to models with real time adaptive controllers.
\end{abstract}




\section{Introduction}

Tracking the boundary of moving objects and the analysis of motion have been intensively studied in the past few years in the context of target tracking [9], [12], image compression and video analysis [22]. A subclass of these issues, and probably the most difficult one, is motion analysis and tracking of non-rigid objects such as the human body and muscle actions [2]. Tracking techniques for such objects include boundary detection via active contour methods [23] and optical flow based motion analysis [13]. Combining these methods, we propose a new real time active contour model for boundary tracking and motion analysis of non-rigid objects.

Boundary tracking based on deformable planar contours, known as snakes, was originally introduced by Terzopoulos et. al. (e.g. [23], [14]). Snakes are deformable contours that move under the infiuence of the image forces, under certain internal deformation constraints. The contour dynamics are given by the Euler-Lagrange equations of motion associated with the contour potential. Using gradient based image potential, for example, results in edge-based deformation forces, leading the contour towards high contrast boundaries; thus performing boundary tracking. Typically, the active contour model suffers from two major problems. The first relates to image clutter which, during tracking of an object, forces the contour towards other image boundaries, and the second problem consists of numerical issues associated with the solution of the equations of motion. Recent work tried to overcome the numerical problems through curve evolution methods [19] and by applying model based boundary repre-

sentation such as splines [8], polygonal approximation [10], and 3-D deformable superquadric models [20].

While the problem of tracking moving objects is continuous in time, the tracking scheme presented in these studies is stationary in its nature, as the contour evolves under the influence of a single image. More recent techniques limit the contour position to areas where there is a change between successive images [10], [21]. This still does not change the static nature of tracking and the approach is limited to stationary cameras. Stepping to the next image 
before convergence or temporary boundary disappearance due to lighting conditions or occlusions, may cause unpredictable effects on the tracking contour. These problems could be prevented if the vision based tracking were treated as a continuous process in time, leading to predictive capabilities through combined space-velocity estimation techniques.

Motion estimation from a sequence of images is based on the apparent motion of brightness pattern in the image, known as optical flow [13]. Many solutions for computing the optical flow have been proposed in the past two decades, among of which include regularization techniques [13], [16], estimation theory based methods [18], and velocity segmentation [1]. These methods are the basis for target tracking, position prediction and 3-D reconstruction of object structure. Incorporating the motion estimation within intensity based contour tracking, we propose a new 2-D tracking scheme in which the contour dynamics are controlled both by the boundary contrast and by the object velocity. This leads to a new tracking method of non-rigid objects with temporal motion estimates and predictive capabilities. The numerical approximation of the equations of motion is also shown to be more stable compared to the original snake model [23].

\section{Preliminaries}

In this section we present the active contour model originally proposed by Terzopoulos et. al. [23], and the principles of optical flow estimation (e.g. [4]).

\subsection{The Active Contour Model}

Consider the closed contour $v(s, t)=(x(s, t), y(s, t))$ for some spatial parametric domain $s \in[0,1]$ and time $t \in[0, \infty)$. Let $v_{s} \triangleq \frac{\partial v}{\partial s}$ and $v_{s s} \triangleq \frac{\partial^{2} v}{\partial s^{2}}$. The Lagrangian energy of the snake, proposed by Terzoupulos et. al. [23], is given by

$$
\frac{1}{2} \int_{0}^{1}\left(w_{1}\left\|v_{s}\right\|^{2}+w_{2}\left\|v_{s s}\right\|^{2}\right) d s+\frac{1}{2} \int_{0}^{1} \mu\left\|v_{t}\right\|^{2} d s+\int_{0}^{1} P(v) d s
$$


The first term in this equation defines the internal deformation energy with $w_{1}(s)$ and $w_{2}(s)$ controlling tension and rigidity, respectively. The second and third terms are the kinetic and potential field energies of the contour. Given the image sample $I(\mathbf{x})$ where $\mathbf{x}=(x, y)$ denote the spatial coordinates, typical potential field energies are functions of image intensity with $P= \pm I(\mathbf{x})$, or contrast, with $P= \pm\|\nabla I(\mathbf{x})\|$, where $\nabla I$ denotes the spatial gradient of $I(\mathbf{x})$. The energy dissipation function which is used to dampen the snake energy (1), so it can converge to a static position, is given by [23]

$$
D\left(v_{t}\right)=\frac{1}{2} \int_{0}^{1} \gamma\left\|v_{t}\right\|^{2} d s .
$$

Assuming the Lagrangian (1) and the above energy dissipation function, the Euler-Lagrange equations of motion are then given by [23]

$$
\mu v_{t t}+\gamma v_{t}-\frac{\partial}{\partial s}\left(w_{1} v_{s}\right)+\frac{\partial^{2}}{\partial s^{2}}\left(w_{2} v_{s s}\right)=-\nabla P(v(s, t)) .
$$

Next we consider the discretization of the snake (3) both in space and time. Consider equidistant sampling of the contour $v(s, \cdot)$ along $s$ with a sampling distance $h(h>0)$, and let $u=\left[u_{1}, \ldots, u_{M}\right]$ be the vector of samples with $u_{i}=v\left(s_{i}, \cdot\right)$ denoting the $\mathrm{i}$-th sample of $v(s, \cdot)$. Using finite difference approximation, the partial derivatives are given by

$$
v_{s}\left(s_{i}\right) \approx\left(u_{i+1}-u_{i}\right) / h \text { and } v_{s s}\left(s_{i}\right) \approx\left(u_{i+1}-2 u_{i}+u_{i-1}\right) / h^{2} \text {. }
$$

Substituting (4) into (3), the discrete version of the contour motion is given by [23]

$$
\mu u_{t t}+\gamma u_{t}+K u=-\nabla P(u)
$$

where the deformation matrix $K=\left[k_{i j}\right]$ satisfies

$$
k_{i j}= \begin{cases}\left(w_{1, i-1}+w_{1, i}\right) / h^{2}+\left(w_{2, i-1}+4 w_{2, i}+w_{2, i+1}\right) / h^{4} & \text { if } i=j, i=0, \ldots, M-1 \\ -w_{1, i} / h^{2}-2\left(w_{2, i}+w_{2, i+1}\right) / h^{4} & \text { if } i=0, \ldots, M-1, j=i+1 \\ -w_{1, j} / h^{2}-2\left(w_{2, j}+w_{2, j+1}\right) / h^{4} & \text { if } j=0, \ldots, M-1, i=j+1 \\ w_{2, i+1} / h^{4} & \text { if } i=0, \ldots, M-1, j=i+2 \\ w_{2, j+1} / h^{4} & \text { if } j=0, \ldots, M-1, i=j+2 \\ 0 & \text { otherwise }\end{cases}
$$


Note that the indices in (6) are modulo $M$. The discretization of (5) in time is based on the central difference approximation

$$
u_{t}(\cdot, t) \approx \frac{1}{2 T}(u(\cdot, t+1)-u(\cdot, t-1)) \text { and } u_{t t}(\cdot, t) \approx \frac{1}{T^{2}}(u(\cdot, t+1)-2 u(\cdot, t)+u(\cdot, t-1))
$$

where $T$ is the sampling interval. A performance analysis of the snake model can be found in $[17]$.

\subsection{Velocity Estimation}

Let $I(\mathbf{x}, t)$ denote the image intensity of the sample at time $t$. Assuming intensity is conserved, i.e. $\frac{d I(\mathbf{x}, t)}{d t}=0$, and a sufficiently small translation between successive images, velocity estimation degenerates into the solution of the gradient constraint equation (e.g. [13])

$$
\nabla I(\mathbf{x}, t) \cdot w+I_{t}(\mathbf{x}, t)=0
$$

where $w=\left[w_{x}, w_{y}\right]^{T}$ is the unknown velocity vector and "." denotes the dot product. $w^{T}$ denotes the transpose of $w$. As the above constraint equation doesn't have a unique solution, further constraints are needed. An example for that is the combined minimization of the gradient constraint equation with a global smoothness term. The optical flow is then computed by minimizing [13]

$$
\int_{D}\left(\frac{1}{2}\left(\nabla I \cdot w+I_{t}\right)^{2}+\frac{\beta^{2}}{2}\|\nabla w\|^{2}\right) d \mathbf{x}
$$

where $D$ is the image domain. If $w$ is assumed to be the result of an affine transformation, i.e.

$$
w=A \mathbf{x}+b
$$

for some real matrix $A$ and vector $b$, it can be shown that $\nabla I \cdot w=C_{\mathrm{x}}^{T} \xi_{w}$, where the row vector $C_{\mathbf{x}}^{T}$ is a function of $\nabla I$ and $\mathbf{x}$ and $\xi_{w}$ is the column vector of the parameters of the affine transformation. Minimizing (9) with $\beta=0$ leads to the solution (e.g. [5])

$$
\xi_{w}=-\left(\int_{D} C_{\mathbf{x}} C_{\mathbf{x}}^{T} d \mathbf{x}\right)^{-1} \int_{D} C_{\mathbf{x}} I_{t} d \mathbf{x}
$$




\section{Combined Space-Velocity Tracking}

The tracking scheme of the deformable contour is space dependent only, as the contour evolves according to the spatial gradients of a static image. This corresponds to proportional (P) control (e.g. [6]). A proportional-integral (PI) control, on the other hand, is known to be more stable and has better tracking characteristics such as zero steady state error for constant velocity inputs (e.g. [6]). Following this result, we propose a new snake model which, in addition to error-dependent position tracking, minimizes the velocity difference between the contour dynamics and the boundary velocity. As this term is unknown in advance, we substitue for it the estimated velocity of the image at the contour position.

\subsection{The Space-Velocity Tracking Model}

Consider the Lagrangian (1) with a time varying potential field function $P(v, t)$. Typically this function corresponds to the space gradient of the temporal image $I(\mathbf{x}, t)$. Let $v^{b}$ and $v_{t}^{b}$ denote the boundary position and velocity, respectively. Generalizing the dissipation function in (2), we propose the velocity-tracking energy dissipation function

$$
D\left(v_{t}, v_{t}^{b}\right)=\frac{\gamma}{2} \int_{0}^{1}\left\|L^{T}\left(v_{t}-v_{t}^{b}\right)\right\|^{2} d s+\frac{\beta}{2} \int_{0}^{1}\left\|\frac{\partial}{\partial s} v_{t}\right\|^{2} d s \quad \gamma>0, \beta>0
$$

where $L$ is a real matrix. The second term represents a smoothness constraint. Assuming the Lagrangian (1), a time varying potential field function $P(v, t)$ and the above dissipation function, the Euler-Lagrange equations of motion of the new snake model are then given by

$$
\mu v_{t t}+\gamma C\left(v_{t}, v_{t}^{b}\right)-\beta \frac{\partial}{\partial s}\left(\frac{\partial}{\partial s} v_{t}\right)-\frac{\partial}{\partial s}\left(w_{1} v_{s}\right)+\frac{\partial^{2}}{\partial s^{2}}\left(w_{2} v_{s s}\right)=-\nabla P(v(s, t), t)
$$

where the velocity control term $C\left(v_{t}, v_{t}^{b}\right)$ satisfies

$$
C\left(v_{t}, v_{t}^{b}\right)=L L^{T}\left(v_{t}-v_{t}^{b}\right)
$$

Note that since the velocity of the object boundary is unknown in advance, we use instead the image velocity estimate at the contour position. Comparing the resulting equation to the 
original model (3), we find the velocity error control term $\gamma L L^{T}\left(v_{t}-v_{t}^{b}\right)$ used for minimizing the error between the contour and the object's velocities, and the velocity smoothness term $-\beta \frac{\partial}{\partial s}\left(\frac{\partial}{\partial s} v_{t}\right)$. As will be shown, the smoothness term also increases the stability of the numerical solution.

One of the major problems in object tracking is boundary disappearance, which may result due to changes in lighting conditions or occlusions. Unlike existing contour models, the proposed approach allows for velocity-based object tracking in the absence of spatial boundary information. The velocity is given by the results of motion estimation prior to boundary disappearance. The tracking model in this case is given by the contour model (13) with $\nabla P(v, t) \equiv 0$. Since image velocity is space dependent, the velocity estimation and prediction is based on a model-based representation of motion, such as the affine transformation (10). This approach allows for velocity estimation based on previous measurements. An estimation method for the velocity parameters is given in the next subsection.

Next we show that while the new model converges to the boundary of an object moving with constant velocity, there is a bias between the solution of the original model and the actual boundary. This bias is proportional to the object velocity and causes "cracks" in the tracking scheme, where parts of the snake stop tracking the boundary and converge to some other objects in the scene. Assuming objects moving at constant velocity in a world with a Lipschitz continuous depth, it can be shown that the image velocity (optical flow) is also Lipschitz continuous , i.e.,

$$
\left\|\mathrm{x}_{t}\left(\mathrm{x}_{1}\right)-\mathrm{x}_{t}\left(\mathrm{x}_{2}\right)\right\| \leq K\left\|\mathrm{x}_{1}-\mathrm{x}_{2}\right\| \text { if }\left\|\mathrm{x}_{1}-\mathrm{x}_{2}\right\| \leq \epsilon
$$

for some $K>0$ and $\epsilon>0$, where $\mathbf{x}_{t}$ is the image velocity (optical flow). The above assumption is reasonable as the image we get is a smoothed digitized version of the real world. The following result describes the tracking properties of the space-decoupled version of (13), i.e., with $\beta=w_{1}=w_{2}=0$. The result clarifies the importance of the proposed velocity control term. 
Theorem 3.1 (Zero Bias Tracking): Consider the system (13) with $\beta=w_{1}=w_{2}=0$ and $\gamma>0$. Assuming constant boundary velocity $v_{t}^{b}$ and the initial state $v(s, 0)$ such that $\left\|v(s, 0)-v^{b}(s, 0)\right\| \leq \epsilon$ for some $\epsilon>0$, then

A. $v(s, t)_{t \rightarrow \infty} \rightarrow v^{b}(s, t)$.

$B$. If $v_{t}^{b}$ in the control term (14) is replaced by the image velocity at the contour, $v_{t}^{i}$, and the image velocity is Lipschitz continuous in the boundary vicinity, then there exist some positive scalars $P$ and $Q$ such that for $0<\gamma<P$ and $0<\mu<Q$ we have $v(s, t)_{t \rightarrow \infty} \rightarrow v^{b}(s, t)$.

C. If $v_{t}^{b} \equiv 0$ in (14), we have $\lim _{t \rightarrow \infty}\left\|v(s, t)-v^{b}(s, t)\right\|>k\left(v_{t}^{b}\right)$ for some positive function $k(\cdot)$.

The proof is given in Appendix A. According to the above result, the contour will converge to the boundary moving at constant velocity if the initial contour is sufficiently close to the boundary of the moving object, and if we have the velocity of the object at the contour position (which could be computed via optical flow techniques). On the other hand, we show that the lack of motion control in the original model causes a bias in the contour position, which could lead to serious tracking problems even in the case of non cluttered environments. Consider the system (13) with $v_{t}^{b}$ replaced by the apparent motion $v_{t}^{i}$ of the image at $v(s, t)$. A special case of interest of this model is when $L=\nabla I(v(s, t))$. In this case the velocity control term (14) satisfies

$$
C\left(v_{t}, v_{t}^{i}\right)=\nabla I\left(\nabla I \cdot v-\nabla I \cdot v_{t}^{i}\right)
$$

As $v_{t}^{i}$ is the apparent velocity, we have, by the optical flow constraint (8), $\nabla I \cdot v_{t}^{i}=-I_{t}$. Substituting this result into (14) and (13), we obtain the optical flow constraint-based tracking model

$$
\mu v_{t t}+\gamma \nabla I\left(\nabla I \cdot v_{t}+I_{t}\right)-\beta \frac{\partial}{\partial s}\left(\frac{\partial}{\partial s} v_{t}\right)-\frac{\partial}{\partial s}\left(w_{1} v_{s}\right)+\frac{\partial^{2}}{\partial s^{2}}\left(w_{2} v_{s s}\right)=-\nabla P(v(s, t), t)
$$

In this model there is no need to estimate the image velocity because the optical flow term provides a measure of the error in velocity estimation. However, this scheme is more sensitive 
to measurements and numerical noise than the model given in (13), as instead of the velocity error, we have a projected version on the direction of $\nabla I$.

\subsection{Boundary Velocity Estimation}

In this section we deal with estimation of the image velocity at the contour. We propose two types of estimation methods: Batch Mode, in which the velocity is estimated independently of the contour dynamics, and Real Time Mode, which uses the temporal velocity estimation error as a control term in the contour dynamics. The proposed methods generalize the parameter estimation techniques in adaptive schemes, such as adaptive control (e.g. [3]), to the case of time-space dependent parameter estimation in nonlinear dynamical systems.

\section{Batch Mode}

Many methods for estimation of the optical flow have been proposed (e.g. [4]). In this work we use the method proposed by Horn and Schunk [13] because it relates directly to the velocity based dissipation proposed in (12). Since we need the image velocity only at the contour position, we use (9) with $D=v(s, t) s \in[0,1]$, and integrate along $s$. The solution to this problem, given by the Euler-Lagrange equation, is

$$
\begin{gathered}
\beta w_{s s}(s, t)=\nabla I(v(s, t))\left(\nabla I(v(s, t)) \cdot w+I_{t}(v(s, t))\right) \\
\text { with } w(0, t)=w(1, t) .
\end{gathered}
$$

where $w=v_{t}^{i}$ is the image velocity at the contour. This problem can be solved using an iterative method similar to the one proposed by Horn and Schunk [13]. Further discussion of the numerical solution is given in the next section.

For the least squares problem (9), we assume no correlation between the velocity parameters at different time points. In our model, however, the image velocity is assumed to be continuous both in time and space, which could be modeled as a slowly time-varying process. This modeling includes also the case of non-rigid objects, in which the motion parameters are assumed to be varying slowly with time. One approach for estimating such slowly time-varying 
parameter, is the least squares method with an exponential "forgetting" of measurements over time (e.g. [3]). Applying this approach, we propose the following velocity estimation objective function

$$
\int_{0}^{1}\left(\frac{1}{2} \int_{0}^{t} \exp -\alpha(t-\tau)\left[\nabla I(v(s, \tau)) \cdot w(s, t)+I_{t}(v(s, \tau))\right]^{2} d \tau+\frac{\beta}{2}\left\|w_{s}\right\|^{2}\right) d s
$$

In this model we assume that the contour parameterization $s$ does not change over time (at least during the effective interval of integration). A discrete version of this model can be

found in [11]. Note that this function also generalizes the energy dissipation function (12). The Euler-Lagrange equation corresponding to (17) is

$$
\left(R(s, t)-\beta \frac{\partial^{2}}{\partial s^{2}}\right) w(s, t)=-P(s, t)
$$

where

$$
R(s, t)=\int_{0}^{t} \exp (-\alpha(t-\tau)) \nabla I \nabla I^{T} d \tau \text { and } P(s, t)=\int_{0}^{t} \exp (-\alpha(t-\tau)) \nabla I I_{t} d \tau
$$

We will show that the discrete version of the velocity estimation (18) can be solved recursively along time. Combining this real time estimation of the image velocity with the velocity control (14) and snake model (13), we obtain a "pseudo" real time tracking model with an optimal velocity control term. "pseudo" refers to the fact that the velocity is estimated independently of the contour dynamics.

\section{Real Time Mode}

Consider (17) as the energy dissipation function for the contour Lagrangian (1). The EulerLagrange equations of motion of the time varying model, are then given by (13) with the velocity control term

$$
C\left(v_{t}\right)=R(t) v_{t}(t)+P(t)
$$

where $R(t)$ and $P(t)$ are given in (19). These terms can be computed recursively according to

$$
\frac{d R(t)}{d t}=-\alpha R(t)+\nabla I(t) \nabla I(t)^{T} \text { and } \frac{d P(t)}{d t}=-\alpha P(t)+\nabla I(t) I_{t}(t)
$$


Note that (20) and (21) are also functions of $s$. In case where we assume no correlation between the velocity parameters at different times $(\alpha \rightarrow \infty)$, the dissipation function (17) coincides with the one proposed by (9) with $D=v(s, t)$ and $s \in[0,1]$. In this case we have $R=\nabla I \nabla I^{T}$ and $P=\nabla I I_{t}$ in $(20)$, and the snake model is the optical flow constraint-based tracking model (15). Compared to (15), the above model is less sensitive to measurements and numerical noise, as the control term averages the optical flow constraint error along time. In case of a rapid change in velocity, however, which could also be caused by depth discontinuities, the above scheme could lead to bias in the control term (20).

\section{Structured Mode}

The velocity estimation schemes proposed in the previous subsections assume no spatial model for the image velocity. The lack of spatial relation between the velocities in different parts of the projected object, could lead to noisy control in the case of the temporary criteria (9), or to a biased one in the case of measurements integration along time, as proposed in (17). A spatial model, on the other hand, will allow for space independent integration of measurements both along space and time, resulting in an improved stability with respect to numerical and measurements noise. This space independent property will further allow for the prediction of the image velocity at the contour and, hence, the prediction of the contour position in the near future. A key point in defining the space model for the velocity is to know the 3-D structure of the imaged object.

Let $(X, Y, Z)$ represent the Cartesian coordinate system of the camera and let $(x, y)$ represent the corresponding coordinates of the image plane, with the $x$ and the $y$ axes parallel to $X$ and $Y$, respectively. The origin of the planar image is given by $(X, Y, Z)=(0,0,1)$. Relative to the camera coordinate system $(X, Y, Z)$, it was shown that the projected velocity $\mathbf{w}=\left[w_{x}, w_{y}\right]^{T}$ of a point undergoing translation with a translation velocity $T=\left(T_{X}, T_{Y}, T_{Z}\right)$ 
and rotation with a rotation velocity $\Omega=\left(\Omega_{X}, \Omega_{Y}, \Omega_{Z}\right)$, satisfies (e.g. [1])

$$
\begin{aligned}
& w_{x}=-\Omega_{X} x y+\Omega_{Y}\left(1+x^{2}\right)-\Omega_{Z} y+\left(T_{X}-T_{Z} x\right) / Z \\
& w_{y}=-\Omega_{X}\left(1+y^{2}\right)+\Omega_{Y} x y+\Omega_{Z} x+\left(T_{Y}-T_{Z} y\right) / Z
\end{aligned}
$$

According to the above result the image velocity is linear in the velocity components. This property can allow for an analytic solution to the velocity estimation problem, if the depth term $Z$ in each of the image points is known. Under the assumption that the imaged region is a planar surface, i.e., $k_{X} X+k_{Y} Y+k_{Z} Z=1$ for some real scalars $k_{X}, k_{Y}$ and $k_{Z}$, the image velocity (22) becomes the following transformation [1]

$$
\begin{aligned}
& w_{x}=a_{1}+a_{2} x+a_{3} y+a_{7} x^{2}+a_{8} x y \\
& w_{y}=a_{4}+a_{5} x+a_{6} y+a_{7} x y+a_{8} y^{2}
\end{aligned}
$$

for some scalars $a_{1}, \ldots, a_{8}$. The affine transformation (10) is an approximation to (23).

Consider the batch mode estimation criteria (17) with the velocity model $(23)((10))$. Since the proposed velocity model is smooth in $\mathrm{x}$, we solve the estimation problem with $\beta=0$. Differentiating (17) with respect to $w$ and equating the resulting function to zero, the optimal velocity parameter vector $\xi_{w}=\left[a_{1}, \ldots, a_{8}\right]^{T}$ satisfies

$$
\xi_{w}(t)=-\left(\int_{0}^{t} \int_{v(s, \cdot)} \exp (-\alpha(t-\tau)) C_{\mathbf{x}} C_{\mathbf{x}}^{T} d s d \tau\right)^{-1} \int_{0}^{t} \int_{v\left(s_{*} \cdot\right)} \exp (-\alpha(t-\tau)) C_{\mathbf{x}} I_{t} d s d \tau
$$

where the row vector $C_{\mathbf{x}}^{T}$ satisfies $\nabla I \cdot w=C_{\mathbf{x}}^{T} \xi_{w}$. In the case where we assume no correlation between the velocity parameters along time $(\alpha \rightarrow \infty)$, the above result coincides with the solution (11).

\section{Implementation Considerations}

In this section we consider the discretization in space and time of the equations of motion of the proposed velocity controlled contours. The continuous models are transformed into simple second order discrete-time equations with nonlinear image-based inputs through conventional finite difference techniques, resulting in low complexity tracking schemes. 


\subsection{Space-Time Discretization}

Consider the space discretization of the equations of motion as proposed in section 2 , and let $u=\left[u_{1}, \ldots, u_{M}\right]$ denote the contour sampling vector. Using the finite difference approximation (4), the discrete version of (13) is given by

$$
\mu u_{t t}+\gamma L L^{T}\left(u_{t}-u_{t}^{b}\right)+\beta D D^{T} u_{t}+K u=-\nabla P(u, t)
$$

where

$$
D=\left[\begin{array}{ccccc}
-1 & 1 & 0 & \ldots & 0 \\
0 & -1 & 1 & 0 . . & 0 \\
\cdot & . & . & \ldots & . \\
1 & 0 & \ldots & \ldots & -1
\end{array}\right]^{T}
$$

is the "derivative" matrix. In this model we have the velocity smoothing term $\beta D D^{T} u_{t}$ in addition to the space smoothing term $K u$. This control further increases the stability of the numerical solution in terms that nearby contour points are prevented from getting too close. As was presented in section 3, a special case of interest of the contour model (13) with $L=\nabla I$, results in the optical-flow constraint-based tracking model (15). Based on (25), the discrete version of this model along space satisfies

$$
\mu u_{t t}+\gamma \nabla I\left(\nabla I \cdot u_{t}+I_{t}\right)+\beta D D^{T} u_{t}+K u=-\nabla P(u, t)
$$

The discretization of time in both models (25) and (27) is performed by the central difference approximation $(7)$.

Many techniques have been proposed for calculating the spatial and time derivatives of images. In this work we use the Sobel operator for approximating the space derivatives and substraction between successive images as a temporal derivative approximation. Prior to the application of these filters we smoothed the images, both along space and time, with a Gaussian filter. An extensive study of derivative approximations and smoothing operations for optical flow estimation can be found in [4], and in [17] for active contours. 


\subsection{Discrete Velocity Estimation}

Consider the solution to the velocity estimation problem (16). Let $\Pi_{x}=\operatorname{diag}\left(I_{x}(u)\right)$ and $\Pi_{y}=\operatorname{diag}\left(I_{y}(u)\right)$, where $\operatorname{diag}(b)$, for some $M$ dimensional vector $b$, is an $M \times M$ diagonal matrix, with the diagonal elements given by $b$. Let

$$
R(t)=C(t) C(t)^{T} \text { and } P(t)=C(t) I_{t}(u, t)
$$

where $C(t)=\left[\begin{array}{ll}\Pi_{x} & \Pi_{y}\end{array}\right]^{T}$, and

$$
\Psi=\left[\begin{array}{ll}
D & 0 \\
0 & D
\end{array}\right]
$$

where $D$ is the derivative matrix (26). Let $w=\left[w_{x}^{i}, w_{y}^{i}\right]^{T}$ be the unknown velocity vector of the image at $u$, where $w_{x}^{i}$ and $w_{y}^{i}$ are the vectors of velocities along the $x$ and $y$ axes, respectively. Substituting (28) and (29) into (16), we obtain (e.g. [18])

$$
\left(R+\beta \Psi \Psi^{T}\right) w=-P I_{t}
$$

Under the condition that $\left(R+\beta \Psi \Psi^{T}\right)$ is invertible, the solution to $(30)$ is $w=-(R+$ $\left.\beta \Psi \Psi^{T}\right)^{-1} P I_{t}$ (e.g. [18]). This problem can also be solved by the iterative Gauss-Seidel method [13].

In the case of the time-varying function (17), the solution to the velocity estimation problem (18) is given by (30) with

$$
R(u, t)=\int_{0}^{t} \exp (-\alpha(t-\tau)) C C^{T} d \tau \text { and } P(u, t)=\int_{0}^{t} \exp (-\alpha(t-\tau)) C I_{t}(u, \tau) d \tau .
$$

Next we will show that, under these conditions, (30) can be solved recursively along time. The proof is based on differentiating both sides of $(30)$ with respect to time.

Lemma 4.1: With $R$ and $P$ given in (31), if $\left(R+\beta \Psi \Psi^{T}\right)$ is invertible for all t, the solution $w$ of (30) satisfies

$$
\frac{d w}{d t}=-Q(t)\left(C(t) e(t)+\alpha \beta \Psi \Psi^{T} w\right)
$$

where $e(t)=\left(I_{t}(u, t)+C^{T}(t) w\right)$ and

$$
\frac{d Q}{d t}=\alpha Q-Q\left(\alpha \beta \Psi \Psi^{T}+C C^{T}\right) Q
$$


Note that $Q=\left(R+\beta \Psi \Psi^{T}\right)^{-1}$. Alternatively one can use the recursive version $(21)$ of $R(t)$ and compute $Q$ for each time. This version is less complex, at least in the memory-space sense, since the matrix $R$ is sparse. Other discrete versions of this result can be found in [11] in the context of optical flow estimation.

\section{Experimental Results}

We demonstrated the performance of the proposed velocity controlled contour model by applying it to real image sequences with both rigid (turning car) and non-rigid (waiving hand) objects. The initial contour lines were generated manually, forming a rough polygonal approximation to the object's boundary. This task could be done automatically via ATR -based methods followed by rough boundary approximation, as the boundary need not be determined exactly.

Prior to the calculation of image gradients and velocities, the image sequences were smoothed, both in space and time, by a Gaussian filter. In the proposed examples we used a fixed Gaussian filter with $\sigma=2$, both in space and time. Spatial derivatives of the images were calculated by applying a simple $3 \times 3$ Sobel operator. The sampling interval along time was defined to be $T=1$.

Contour parameters: We used the contour models with a spatial sampling distance $h=5$ (pixels) and with $\gamma=\mu=2$. The deformation parameters $w_{1}$ and $w_{2}$ were set to constant values. More complex techniques define the values of $w_{1}$ and $w_{2}$ to be nonlinear functions of the distance between nearby points [17]. In our model, however, we found the velocity control to have similar smoothness effects, making these extensions unnecessary. The potential energy function $P=-\|\nabla I(\mathrm{x})\|$ and its directional derivatives were computed using a simple $3 \times 3$ Sobel operator. These operations were made only in the vicinity of the contour.

Velocity estimation: A key distinction between conventional optical fiow estimation in images (e.g. [4]) and our boundary velocity estimation, is that the latter is performed only at the contour, resulting in reduced computational effort. The time and spatial derivatives of 
the images were calculated by a simple substraction and by applying a $3 \times 3$ Sobel operator, respectively. These calculations were made only in the vicinity of the contour. As was noted, prior to the application of these filters, the image sequence was smoothed, both along space and time, with a Gaussian filter. The resulting time and spatial derivatives were used to calculate the velocity components, as proposed in section 4 .

Image sequences: The tracking schemes were demonstrated on the following image sequences:

A. Hamburg taxi sequence: A street scene with moving cars (Figure 1). The tracking scheme was applied to the taxi turning at the corner. In this case, due to turning, the taxi boundary contour has thought of non-rigid behavior. The sequence is composed of 20 frames sampled at video rate with 1:3 decimation along time (in order to increase the speed of the car).

B. Office scene: An office scene with the author waiving his hand under nonuniform lighting conditions (Figure 2). The scene includes image clutter such as shadows, shelves, books and a computer monitor. The sequence is composed of 20 images sampled at video rate.

Tracking results: The results of tracking the boundary of the turning car are presented in Figures 1(a)-(e). The initial frame and the corresponding initial contour, are given in Figure 1(a). The tracking results of the original model after 19 frames, and those of the proposed velocity controlled models, are presented in Figure 1(b) and in Figures 1(c)-(e), respectively. In the presence of image motion in cluttered environments, the original model is inferior to the velocity controlled model. This result is mainly due to the bias property of the original model, and the inability to incorporate the velocity measurements in the tracking scheme. Note that in our model we do not use any prior knowledge such as boundary and clutter models. Figures 1 (c)-(d) show the results of the batch mode model (25) with the affine velocity model (10) and with the regularization based velocity estimation given by the solution of (30) with $\beta=30$. We found both schemes to lead to similar tracking results. The results of the optical flow constraint-based model (27), shown in Figure 1(e), exhibit slightly larger tracking errors. 
Figures 2(a)-(e) show tracking of the waiving hand. While the original model (Figure 2(b)) is "trapped" by image clutter, e.g. the shelves and the monitor boundary, the new tracking models perform very well ( Figures 2(-c)-(e)). Compared to the batch mode models (Figures 2(c)-(d)), we find again that the optical flow constraint-based model has slightly larger tracking errors (Figure 2(e)).

In Figures 3(a)-(b) we study the results of tracking with Structured Mode motion estimation. Figure 3(a) shows the tracking results of (25) with the affine velocity model (10). The velocity parameters are estimated using (11). The results with the motion model (23) and with parameter estimation via integration of measurements along time (24), are given in Figure 3(b). The time integration was computed via simple discrete sum approximation and with $\exp (-\alpha T)=0.5$. In this case, motion estimation based on a single image sample was found to be too noisy due to the large number of parameters. Comparing Figures $3(a)$ and 3 (b), we find that combining a complex velocity model with measurements integration along a finite time interval results in improved tracking performance. Note that the error in the velocity estimation increases with the complexity of the velocity model.

One of the major advantages of the proposed approach is the ability to predict the position of non-rigid boundaries. This property is demonstrated in Figure 4 where we predicted the boundary position in the 19-th frame, based only on the first eight frames. The contour position in the 8 -th frame is given by the dashed line. The prediction was based on the Structured Mode velocity model (23) with parameter estimation based on (24). 

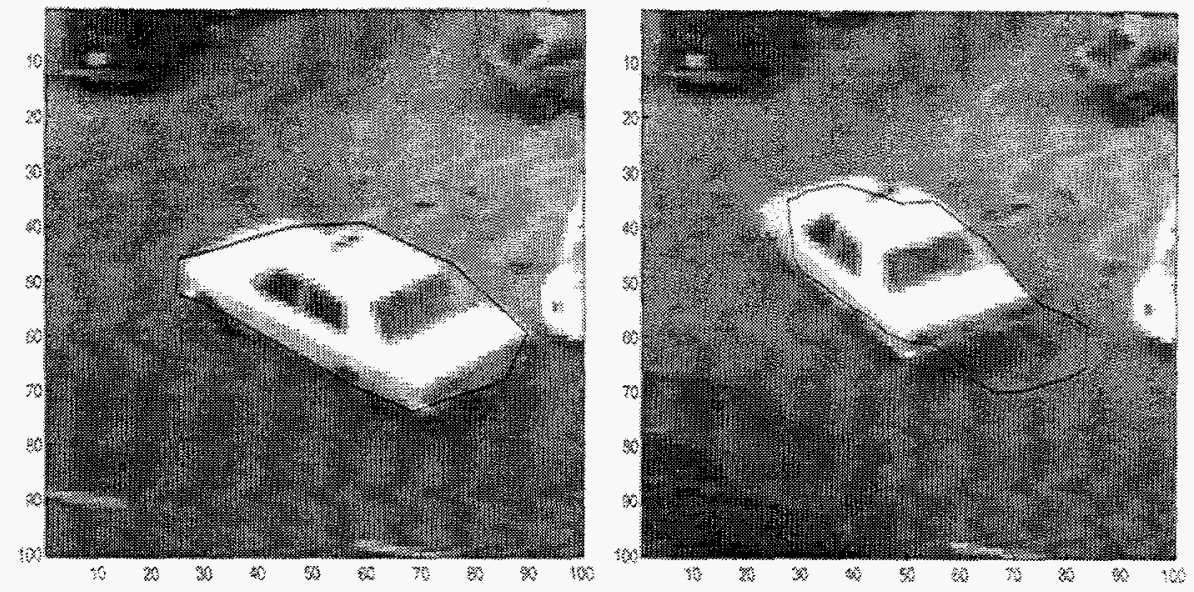

(a)

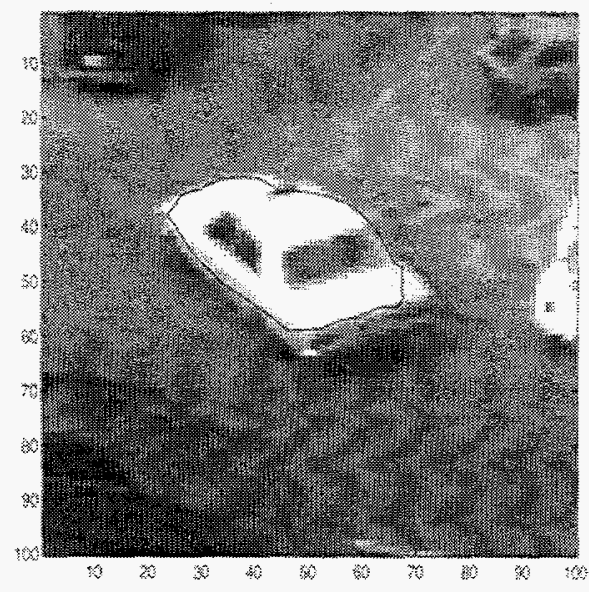

(d)

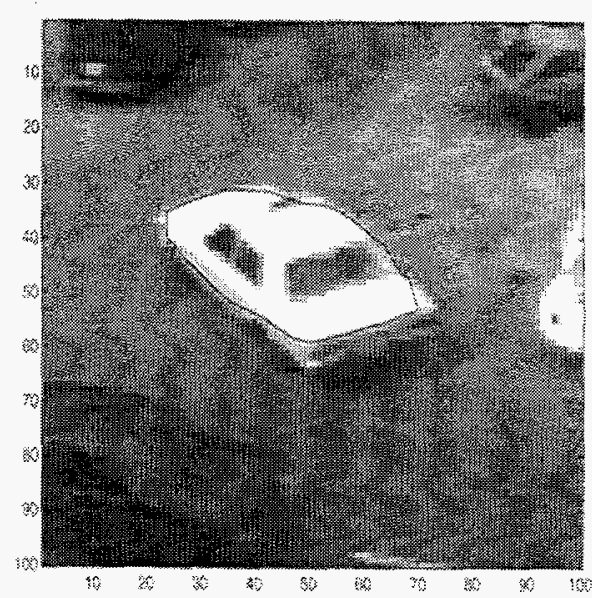

(c)

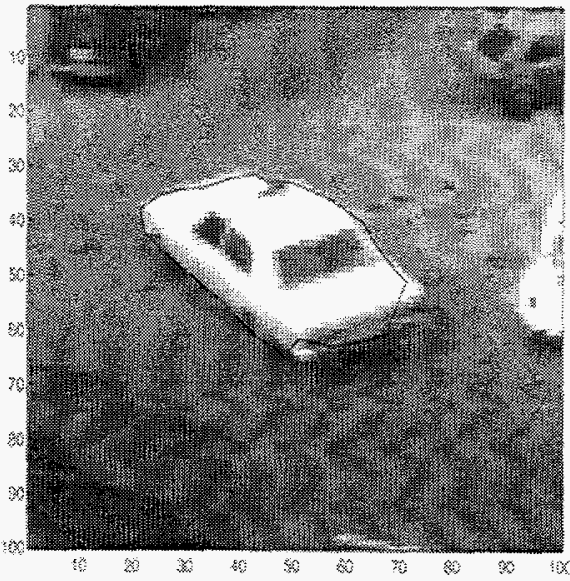

(e)

Figure 1: Tracking results of the turning car (after 19 frames): (a) the initial frame with the initial contour, (b) original snake model, (c) batch-mode model with affine velocity, (d) batch-mode model with regularization based velocity estimation, (e) optical flow constraintbased model. 


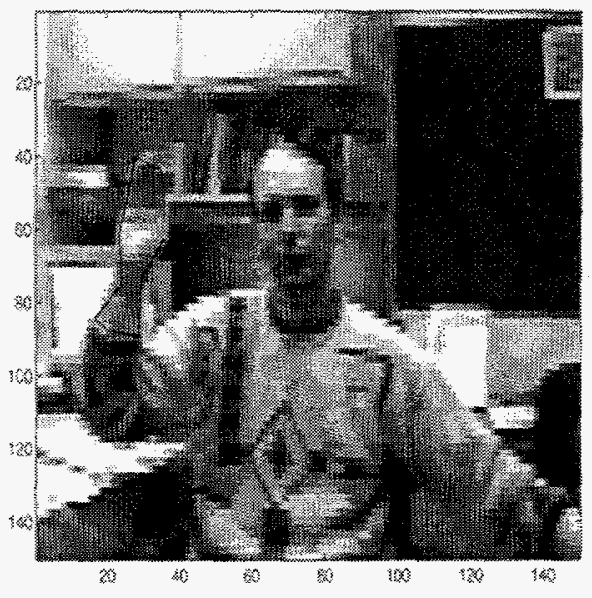

(a)

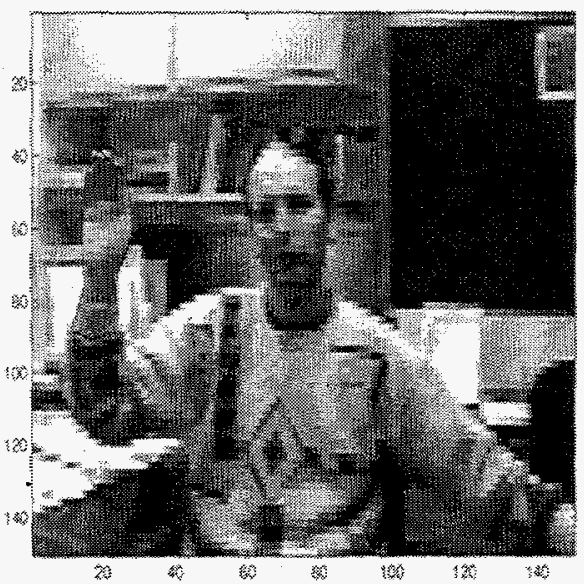

(d)

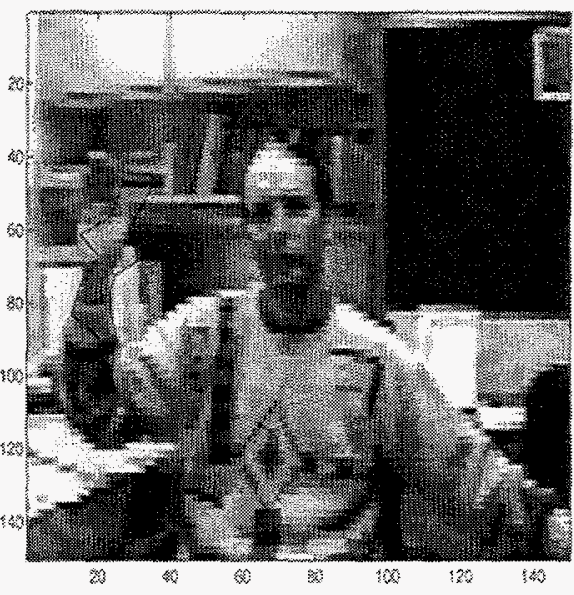

(b)

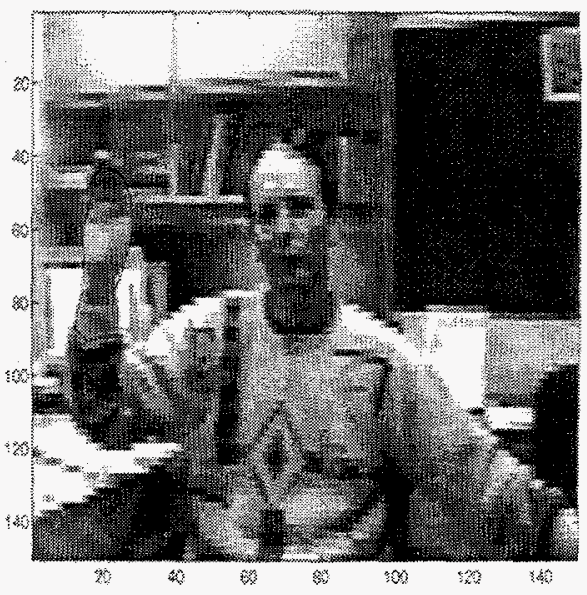

(c)

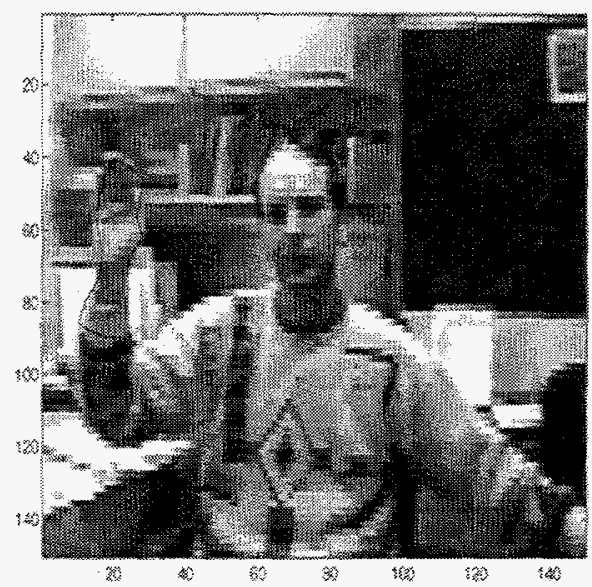

(e)

Figure 2: Tracking results of the waiving hand (after 19 frames): (a) the initial frame with the initial contour, (b) original snake model, (c) batch-mode model with affine velocity, (d) batch-mode model with regularization based velocity estimation, (e) optical flow constraintbased model. 


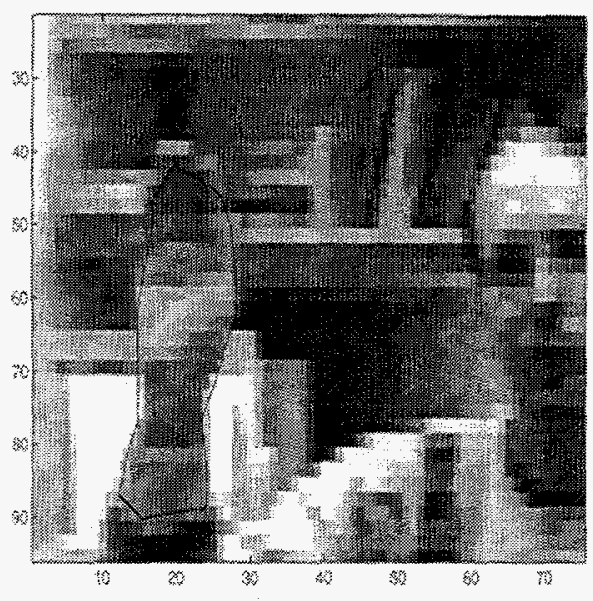

(a)

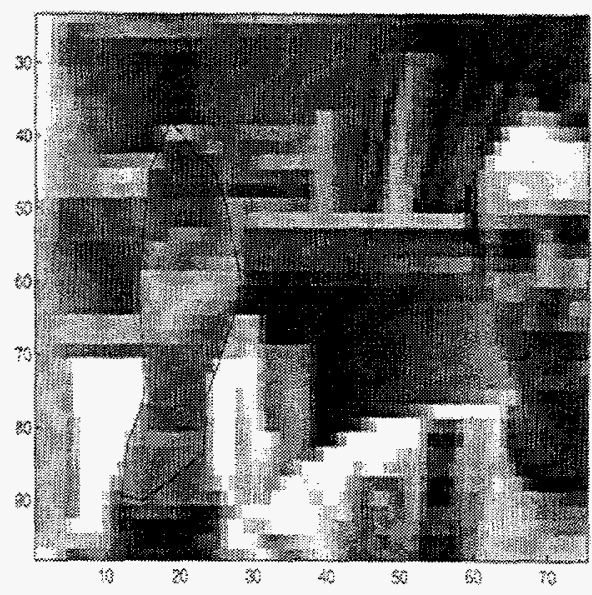

(b)

Figure 3: Tracking results with Structured Mode velocity estimation: (a) affine velocity model, (b) velocity model (23) and parameter estimation based on the measurements integration along time (24).

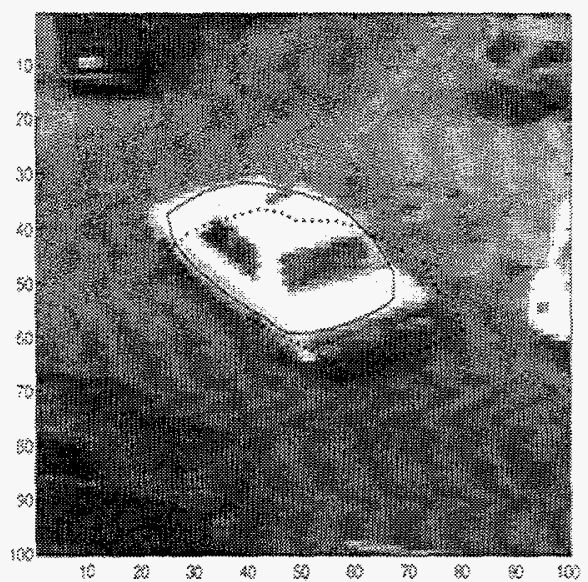

(a)

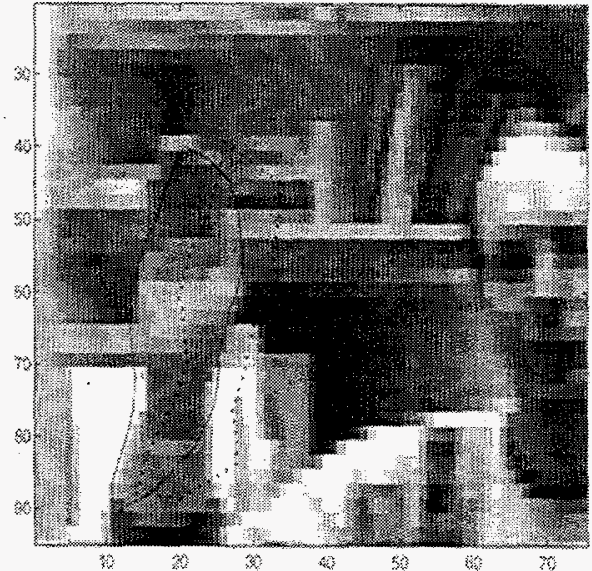

(b)

Figure 4: Boundary prediction in the 19-th frame based on the first eight frames. The dashed line represents the contour position in the 8-th frame. 


\section{Concluding Remarks}

We have described a new class of active contour models which result from applying a velocity control to the class of elastodynamical contour models, known as snakes. We showed that this model allows for unbiased real-time tracking of an object in a video image sequence, provided that the initial contour is sufficiently close to the actual boundary. This demand can be relaxed by applying inflation forces on the contour as proposed by [7], [19]. The unbiased result is in contrast to the original model which is proven to be biased by the object motion, resulting in high sensitivity to clutter and numerical noise. Based on the proposed control approach, we developed a new class of real time tracking contours, varying from models with batch-mode control estimation, to models with real time adaptive controllers. The proposed tracking scheme was applied to boundary tracking of both rigid and non-rigid objects, resulting in unbiased tracking and robustness to image clutter and numerical noise. The velocity-based boundary prediction of non-rigid boundaries was also shown to have good results.

\section{Acknowledgments}

This research was performed at the Center for Engineering Systems Advanced Research, Oak Ridge National Laboratory. Funding provided by the Engineering Research Program of the Office of Basic Energy Sciences under contract DE-AC05-96OR22464 with Lockheed Martin Energy Research, Corp. The author thanks Dr. Jacob Barhen, head of CESAR at ORNL. 


\section{References}

[1] G. Adiv, Determining three-dimensional motion and structure from optical flow generated by several moving objects, IEEE Trans. Pattern Anal. and Machine Intell. PAMI 7(4), 1985, pp. 384-401.

[2] J.K. Aggarwal, Q. Cai, W. Liao and B. Sabata, Articulated and elastic non-rigid motion: A review, in Proc. of IEEE Computer Society Workshop on Motion of NonRigid and Articulated Objects, pp. 16-22, Austin, TX, 1994.

[3] K.J. Astrom and B. Wittenmark, Adaptive Control, Addison-Wesley, 1995.

[4] J.L. Barron, D.J. Fleet and S.S. Beauchemin, Performance of optical flow techniques, Int. J. of Computer Vision 12(1), 1994, pp. 43-77.

[5] J.R. Bergen, P.J. Burt, R. Hingorani and S. Peleg, A three-frame algorithm for estimating two-component image motion, IEEE Trans. Pattern Anal. and Machine Intell. 14(9), 1992, pp. 886-896.

[6] W.L. Brogan, Modern Control Theory, Prentice Hall, Englewood Clifs, New Jersey, 1991.

[7] L.D. Cohen, On active contour models and balloons, CVGIP 53(2), 1991, pp. 211-218.

[8] R. Curwen and A. Blake, Dynamic contours: Real-time active splines, in Active Vision (A. Blake and A. Yuille Eds.), pp. 39-58, MIT Press, 1992.

[9] M.P. Dubuisson and A.K. Jain, Contour extraction of moving objects in complex outdoor scenes, Int. J. Computer Vision 14, 1995, pp. 83-105.

[10] M.P. Dubuisson, S. Lakshmanan and A.K. Jain, Vehicle segmentation and classification using deformable templates, IEEE Trans. Pattern Anal. and Machine Intel. 18(3), 1996, pp. 293-308. 
[11] M. Elad and A. Feuer, Recursive optical flow estimation - Adaptive filtering approach, EE PUB N. 1009, Technion IIT, 1996.

[12] C. Harris, Tracking with rigid models, in Active Vision (A. Blake and A. Yuille Eds.), pp. 59-74, MIT Press, 1992.

[13] B.K.P. Horn and B.G. Schunk, Determining optical flow, Artificial Intelligence 17, 1981, pp. 185-204.

[14] M. Kass, A. Witkin and D. Terzopoulos, Snakes: Active contour models, Int. J. Computer Vision 1(4), 1987, pp. 321-331.

[15] H.K. Khalil, Nonlinear Systems, Prentice Hall, Upper Saddle River, NJ, 1996.

[16] A. Kumar, A.R. Tannenbaum and G.J. Balas, Optical flow: A curve evolution approach, IEEE Trans. Image Processing 5(4), 1996, pp. 598-610.

[17] F. Leymarie and M.D. Levine, Tracking deformable objects in the plane using an active contour models, IEEE Trans. Pattern Anal. and Machine Intell. 15(6), 1993, pp. 617-634.

[18] M.R. Luettgen, W.C. Karl and A.S. Willsky, Efficient multiscale regularization with applications to the computation of optical flow, IEEE Trans. Image Processing $3(1)$, 1994, pp. 41-64.

[19] R. Malladi, J.A. Sethian and B.C. Vemuri, Shape modeling with front propagation: A level set approach, IEEE Trans. Pattern Anal. and Machine Intell. 17(2), 1995, pp. 158-175.

[20] D. Metaxas and D. Terzopoulos, Shape and nonrigid motion estimation through physics-based sythesis, IEEE Trans. Pattern Anal. and Machine Intell. 15(6), 1993, pp. 580-591. 
[21] S. Rowe and A. Blake, Statistical mosaics for tracking, Image and Vision Computing 14(8), 1996, pp. 549-564.

[22] H.S. Sawhney and S. Ayer, Compact representations of videos through dominant and multiple motion estimation, IEEE Trans. Pattern Anal. and Machine Intell. 18(8), 1996, pp. 814-830.

[23] D. Terzopoulos and R. Szeliski, Tracking with kalman snakes, in Active Vision (A. Blake and A. Yuille Eds.), pp. 3-20, MTT Press, 1992. 


\section{Appendix A: Proof of the convergence theorem}

Theorem 3.1 (Zero Bias Tracking): Consider the system (13) with $\beta=w_{1}=w_{2}=0$ and $\gamma>0$. Assuming constant boundary velocity $v_{t}^{b}$ and the initial state $v(s, 0)$ such that $\left\|v(s, 0)-v^{b}(s, 0)\right\| \leq \epsilon$ for some $\epsilon>0$, then

A. $v(s, t)_{t \rightarrow \infty} \rightarrow v^{b}(s, t)$.

B. If $v_{t}^{b}$ in the control (14) is replaced by the image velocity at the contour, $v_{t}^{i}$, and the image velocity is Lipschitz continuous in the boundary vicinity, then there exist some positive scalars $P$ and $Q$ such that for $0<\gamma<P$ and $0<\mu<Q$ we have $v(s, t)_{t \rightarrow \infty} \rightarrow v^{b}(s, t)$.

C. If $v_{t}^{b} \equiv 0$ in (14), we have $\lim _{t \rightarrow \infty}\left\|v(s, t)-v^{b}(s, t)\right\|>k\left(v_{t}^{b}\right)$ for some positive function $k(\cdot)$.

Proof: The following result is based on analyzing the convergence properties of the linearized version of (13) with $\beta=w_{1}=w_{2}=0$. The system performance is then analyzed using Lyapunov theory (e.g. [15]). We shall employ the following definitions: The function $w: \mathbb{R}^{n} \rightarrow \mathbb{R}$ is positive definite if $w(0)=0$ and $w(x)>0$ for $x \neq 0$. The matrix $M=\left[m_{i j}\right]$ is positive definite if it is symmetric, i.e. $m_{i j}=m_{j i}$, and all the eigenvalues have positive real parts (it can be shown that the eigenvalues are real). A matrix $A$ is said to be stable if all the eigenvalues of $A$ have negative real parts. We shall denote the i'th eigenvalue of $A$ by $\lambda_{i}(A)$. The absolute value of the eigenvalue with the largest absolute value shall be denoted by $\lambda_{\max }(A)$ and the smallest one by $\lambda_{\min }(A)$. The matrix $I$ denotes the identity matrix.

Consider the initial state $v(s, 0)$ such that $\left\|v(s, 0)-v^{b}(s, 0)\right\| \leq \epsilon$ for some sufficiently small $\epsilon$. Assuming the image moving with a constant velocity, the potential field field function can be approximated by

$$
P \approx P_{0}+\frac{1}{2}\left(v-v^{b}\right)^{T} M\left(v-v^{b}\right)
$$


for some positive definite matrix $M$. We shall also assume that $L=I$ in (14). Substituting $P$ into (13), the linearized version of (13) about $v^{b}$ is then

$$
\mu v_{t t}+\gamma\left(v_{t}-v_{t}^{b}\right)+M\left(v-v^{b}\right)=0
$$

Let $y=v-v^{b}$. Since $v_{t}^{b}$ is assumed to be constant, it follows that $\dot{y}=v_{t}-v_{t}^{b}$ and $\ddot{y}=v_{t t}$ where $\dot{y}$ also denote the derivative along time. Substituting $y$ into (33) with $y_{1}=y, y_{2}=\dot{y}_{1}$ and $\mathrm{y}=\left[y_{1}, y_{2}\right]^{T}$, we obtain

$$
\dot{\mathrm{y}}=A \mathrm{y} \quad \text { where } \quad A=\left[\begin{array}{cc}
0 & I \\
-\frac{1}{\mu} M & -\frac{\gamma}{\mu} I
\end{array}\right] \text {. }
$$

The matrices $I$ and 0 are $2 \times 2$ identity and zero matrices, respectively. Assuming $\mu>0$ and $\gamma>0$, it can be shown that $A$ is stable iff $M$ is positive definite. This follows from the result that for each eigenvalue of $M, \lambda_{i}(M)$, there exist two corresponding eigenvalues of $A$, given by

$$
\lambda_{12}^{i}=\frac{-\gamma \pm \sqrt{\gamma^{2}-4 \lambda_{i}(M) \mu}}{2 \mu} .
$$

Since $A$ is stable, it follows that $\mathbf{y}_{t \rightarrow \infty} \rightarrow 0$ in (34), and by Lyapunov theorem (e.g. Theorem 3.7 p.127 [15]), $\mathbf{y}_{t \rightarrow \infty} \rightarrow 0$ also in the original model (13) provided the initial state $\mathrm{y}(0)$ is sufficiently small. Hence, the assertion of statement A follows.

If $v_{b}^{t}$ is set to zero in the control term (14), the linearized version of (13) about $v^{b}$ is then

$$
\dot{\mathbf{y}}=A \mathrm{y}+\left[\begin{array}{ll}
0 & -\frac{\gamma}{\mu} v_{t}^{b}
\end{array}\right]^{T} \text {. }
$$

Since $A$ is stable, it can be shown that $y=y_{1} \rightarrow-\gamma M^{-1} v_{t}^{b}$. Since the linearized system is biased, it follows that the original system has also the same property. Hence, the assertion of statement $\mathrm{C}$ follows.

Next we prove statement B. Using (34) and the control term (14) with $L=I$ and $v_{t}^{b}=v_{t}^{i}$, the linearized version of (13) about $v^{b}$ is then given by

$$
\dot{\mathrm{y}}=A \mathrm{y}+\left[\begin{array}{ll}
0 & -\frac{\gamma}{\mu}\left(v_{t}^{b}-v_{t}^{i}\right)
\end{array}\right]^{T} .
$$


We shall prove the convergence of (35) using Lyapunov theory. Given a positive definite function $w(\mathbf{y})$, if the derivative of $w(\mathbf{y})$ along the solution of $(35)$ is negative definite, then the origin of (35) is asymptotically stable (e.g. Theorem 3.1 p.100 [15]). Let $w(y)=\mathbf{y}^{T} P \mathbf{y}$ be a scalar function where the matrix $P$ is given by the solution of the Lyapunov matrix equation

$$
P A+A^{T} P=-Q \text { where } Q=\left[\begin{array}{cc}
-\frac{2}{\mu} M^{2} & 0 \\
0 & -2 M
\end{array}\right] .
$$

Since $A$ is stable and $Q$ is positive definite, it follows, by the Lyapunov matrix equation theorem (e.g. Theorem 3.6 p.123 [15]), that $P$ is positive definite. Hence, $w(y)$ is a positive definite function. Further more, substituting $Q$ and $A$ into (36), it can be shown that

$$
\text { for }\left[\begin{array}{cc}
P_{1} & P_{12} \\
P_{12}^{T} & P_{2}
\end{array}\right]
$$

we have

$$
P_{12}=M \quad \text { and } \quad P_{2}=\frac{2 \mu}{\gamma} M .
$$

Using (35), (36) and (37), the derivative of $w(\mathbf{y})$ along the solution of (35) is then given by

$$
\frac{d w(\mathbf{y})}{d t} \equiv \nabla W \cdot \dot{\mathbf{y}}=-\frac{2}{\mu} y_{1}^{T} M^{2} y_{1}-2 y_{2}^{T} M y_{2}+2\left(y_{1}^{T} M+y_{2}^{T}\left(\frac{2 \mu}{\gamma} M\right)\right)\left(-\frac{\gamma}{\mu}\left(v_{t}^{b}-v_{t}^{i}\right)\right) .
$$

Since the image velocity is Lipschitz continuous, it follows that $\left\|v_{t}^{b}-v_{t}^{i}\right\| \leq K\left\|y_{1}\right\|$. From standard norm inequalities, we obtain

$$
\frac{d w(\mathbf{y})}{d t} \leq 2\left[-\left(\frac{1}{\mu} \lambda_{\min }^{2}(M)-\lambda_{\max }(M) K \frac{\gamma}{\mu}\right)\left\|y_{1}\right\|^{2}-\lambda_{\min }(M)\left\|y_{2}\right\|^{2}+2 \lambda_{\max }(M) K\left\|y_{1}\right\|\left\|y_{2}\right\|\right] .
$$

Since the above equation is quadratic in $\left\|y_{1}\right\|$ and $\left\|y_{2}\right\|$, necessary and sufficient conditions for the right hand side of (39) to be negative definite are

$$
\frac{1}{\mu} \lambda_{\min }^{2}(M)-\lambda_{\max }(M) K \frac{\mathcal{Y}}{\mu}>0
$$

and

$$
\lambda_{\max } K \leq \sqrt{\left(\frac{1}{\mu} \lambda_{\min }^{2}(M)-\lambda_{\max }(M) K \frac{\gamma}{\mu}\right) \lambda_{\min }(M)} .
$$


From the above inequalities it follows that the right hand side of (39) is negative definite iff

$$
\gamma<\frac{\lambda_{\min }^{2}(M)}{K \lambda_{\max }(M)} \text { and } \mu \leq \frac{\lambda_{\min }(M)}{\lambda_{\max }(M) K}\left(\frac{\lambda_{\min }^{2}(M)}{\lambda_{\max }(M) K}-\lambda\right) \text {. }
$$

Under the above conditions, it follows that derivative of $w(\mathbf{y})$ along the solution of (35) is negative definite, and hence the origin is asymptotically stable. This result proves the stability property of the original system (13) (e.g. Theorem 3.7 p. 127 [15]) and hence the convergence of $v$ to $v^{b}$, provided the initial state is sufficiently close to $v^{b}$. Note that the Lipschitz property of the image velocity implies that $\left\|v_{t}(\cdot, 0)-v_{t}^{b}(\cdot, 0)\right\| \leq K \varepsilon$ if $\| v(\cdot, 0)-$ $v^{b}(\cdot, 0) \| \leq \varepsilon$. Hence the assertion of statement B follows. 


\section{INTERNAL DISTRIBUTION}

1-5. J. Barhen

6. C. W. Glover

7. W. C. Grimmell

8. H. E. Knee

9. E. M. Oblow

10. C. E. Oliver

11-15. L. E. Parker

16-20. N. Peterfreund

21. F. G. Pin

22. V. Protopopescu
23. N. S. Rao

24. D. B. Reister

25-29. R. F. Sincovec

30. M. S. Smith

31. E. C. Uberbacher

32. M. A. Unseren

33-37. CSMD Reports Office

38. Laboratory Records - RC

39. Document Reference Section

40. Central Research Library

41. ORNL Patent Office

\section{EXTERNAL DISTRIBUTION}

42-43. Office of Scientific and Technical Information, P.O. Box 62, Oak Ridge, TN 37831.

44. Dr. Fred Aminzadeh, UNOCAL, 14141 SW Freeway, Suite 301-225, Sugarland, TX 77478.

45. Dr. John Baillieul, Aerospace \& Mechanical Engineering Department, Boston University, 110 Cummington Street, Boston, MA 02215.

46. Dr. John Blair, JBX Technologies, 25 Moore Road, Wayland, MA 01778.

47. Dr. Roger W. Brockett, Harvard University, Piece Hall, 29 Oxford Street, Cambridge, MA 02138.

48. Dr. Oscar P. Manley, 13212 Wye Oak Drive, Gaithersburg, MD 20878.

49. Dr. Robert E. Price, ER-15, Office of Basic Energy Sciences, Department of Energy, Washington, DC 20585.

50. Dr. Charles R. Weisbin, Robotics and Mars Exploration Technology Program, Jet Propulsion Laboratory, 4800 Oak Grove Drive, Pasadena, CA 91109. 